\title{
Accurate RF Voltage Measurements Using a Sampling Voltage Tracker
}

\author{
T. MICHAEL SOUDERS, MEMBER, IEEE, AND PAUL S. HETRICK
}

\begin{abstract}
The RF voltage measurement capability of an equivalenttime sampling system has been investigated over the frequency range of $1-100 \mathrm{MHz}$. The system is easily calibrated from step response measurements, independent of thermal transfer standards. Comparison measurements made with NBS-calibrated thermal converters show agreement generally within the stated uncertainties presently provided by NBS for such calibrations. The system offers several advantages over conventional thermal transfer techniques: ac/dc transfers are not required, loading and transmission line problems are reduced, and direct measurement of voltages from $2 \mathrm{~V}$ to as low as $10 \mathrm{mV}$ are possible. In addition, other waveform characteristics are readily obtained, e.g., average and peak values, harmonic distortion, etc.
\end{abstract}

\section{INTRODUCTION}

$\mathrm{R}^{\mathrm{r}}$ F VOLTAGES are generally measured using thermal transfer, average responding (rectifying), or peak responding measurement techniques. The latter two approaches, while offering conveniences of use, are of limited value when measuring nonsinusoidal waveforms. On the other hand, instruments incorporating thermal transfer devices (vacuum thermocouples, solid-state thermal converters, thermistors, etc.) offer good rms measurement accuracy even for highly distorted waveforms. Unfortunately, they generally suffer from several other limitations:

1) an ac/dc transfer is usually required to achieve reasonable accuracy,

2) thermoelements are difficult to protect from overload, and are subject to burnout,

3) input impedances are low, causing loading and impedance matching problems,

4) the dynamic measurement range of thermal transfer devices is limited because of the square law heating characteristic,

5) measurement times are relatively long because of long thermal time constants.

In this work, we present an alternative measurement approach, based on an equivalent-time sampling technique. The technique overcomes many of the limitations noted above, while achieving accuracies comparable to those realized with thermal transfer instruments.

Manuscript received June 10, 1988. This work was supported by Sandia National Laboratories.

The authors are with the Electrosystems Division, National Bureau of Standards, Gaithersburg, MD 20899.

IEEE Log Number 8825620.

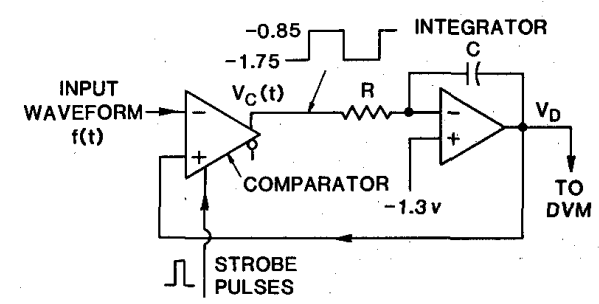

Fig. 1. Basic circuit of the sampling voltage tracker (SVT).

\section{Sampling Voltage Tracker (SUT)}

The new measurement system, called a sampling voltage tracker (SVT), has previously been studied by the authors and others, primarily as a tool for fast time-domain measurements of parameters such as settling time [1]-[3]. The SVT is an equivalent-time sampling and digitizing system which uses a high speed latched IC analog comparator as the sampler. As illustrated in Fig. 1, the latched comparator is operated in a feedback loop to repetitively sample the input signal at a given instant on the waveform and generate a dc level equal to the average sampled voltage. For signal frequencies up to $5 \mathrm{MHz}$, the strobe pulse occurs once each period; above $5 \mathrm{MHz}$ the strobe pulse occurs every two or more periods such that the strobe pulse rate does not exceed $5 \mathrm{MHz}$. Digitization is accomplished by measuring the dc level with a precision DVM. The process is repeated at successive delays determined by a precision time delay generator, until the entire waveform of interest has been characterized. The DVM typically integrates the output for $0.167 \mathrm{~s}$; therefore, each sampled instant on the input waveform is observed a minimum of 167.000 times for input frequencies of $1 \mathrm{MHz}$ or greater. The complete SVT system based on the IEEE488 bus, is shown in Fig. 2.

The comparator and integrator circuits are mounted in a small pod which is connected by an umbilical cable to the other system components. This configuration permits the sampler to be connected directly to the output of the source of voltage to be measured, eliminating the need for connecting cables. The comparator is physically within $1 \mathrm{~cm}$ of the type- $N$ input connector and has an input impedance of $60 \mathrm{k} \Omega$ or greater, shunted by about $2 \mathrm{pF}$. The input can be terminated in $50 \Omega$ at the comparator, if desired. These features are intended to minimize errors due to transmission line effects. For this paper, several different comparator models have been studied; test re-

U.S. Government work not protected by U.S. copyright 


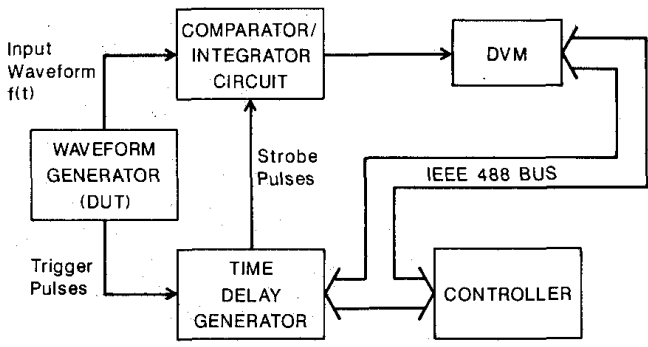

Fig. 2. Block diagram of a complete SVT system.

sults from two of these will be reported, designated SVT 4 and SVT 5. The principal differences between the two are their equivalent bandwidths (approximately 650 and $750 \mathrm{MHz}$, respectively), their bias currents, and their "thermal tails."

RMS voltages are measured by computing the square root of the mean of the squares of the samples over one or an integral number of periods of the input signal. The equivalent sampling rate of the SVT is as high as 100 $\mathrm{GHz}$. With the bandwidths given above, harmonically distorted waveforms can still be accurately measured at fundamental frequencies up to $100 \mathrm{MHz}$. The total harmonic distortion of the SVT ranges from less than 0.1 percent at $1 \mathrm{MHz}$ to about 1 percent at $100 \mathrm{MHz}$ [3], values which contribute negligible error (to an rms measurement) for all but the most seriously distorted waveforms. The rms noise measured with no applied signal is less than $10 \mu \mathrm{V}$. The offset voltage is readily measured with the input terminals shorted and software corrections are then applied.

\section{Gain Corrections Via Step Response MEASUREMENTS}

Small errors in the SVT gain over the frequency range of interest, i.e., deviations from unity, can be estimated from response measurements using a fast rise time, fast settling input step [4]. The step is sampled with an equivalent-time sampling rate sufficient to give negligible aliasing errors over the frequency range of interest. The discrete impulse response is then computed from the step response data, and the frequency magnitude spectrum is obtained by Fourier transform of the impulse response. Gain corrections are computed from the magnitude spectrum. Fig. 3 shows the results from such a measurement of SVT 4. Fig. 3(a) shows the last 4 percent of the step response. The frequency response computed from this data is given in Fig. 3(b). The lower trace of Fig. 3(b) is the frequency response of the SVT to the actual input step; the upper trace is the response of the SVT itself, estimated by deconvolving the input step from the measured SVT response. (The actual function to be deconvolved was estimated from rise-time measurements of the input step, and assumed a single pole response.) The step generator used for these measurements is an improved, commercial version of the NBS Reference Flat Pulse Generator [5]. Its step amplitude is $0.5 \mathrm{~V}$ and the manufacturer specified
STEP RESPONSE (LAST $4 \%$ )

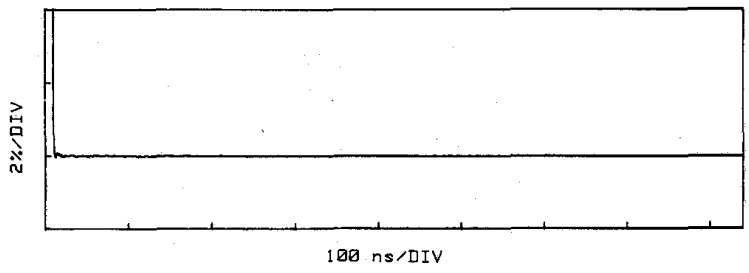

(a)

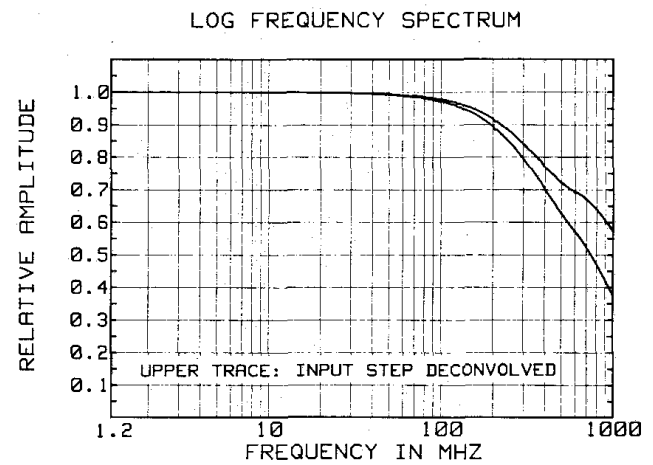

(b)

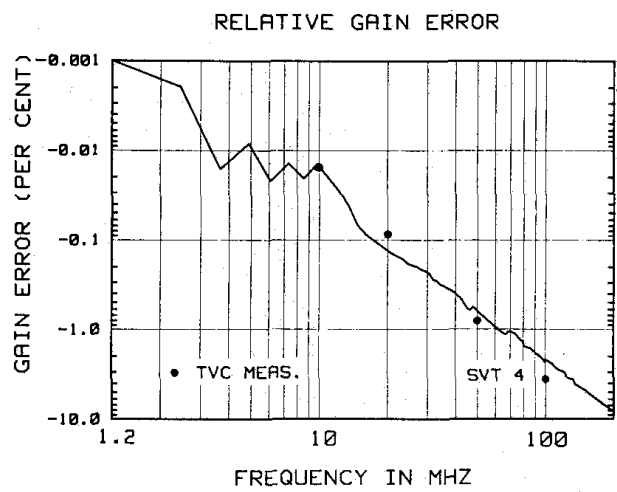

(c)

Fig. 3. Gain corrections from step response measurements, for SVT 4. (a) Showing last 4 percent of step response. (b) Frequency response computed from step response data. (c) Gain error relative to first spectral line at $1.2 \mathrm{MHz}$. The TVC measurement at $1.2 \mathrm{MHz}$ is not plotted in (c); the value is +0.01 percent.

rise time (transition duration) is $397 \mathrm{ps.} \mathrm{Fig.} \mathrm{3(c)} \mathrm{gives} \mathrm{the}$ gain error relative to the first spectral line at $1.2 \mathrm{MHz}$, computed from the deconvolved spectrum of Fig. 3(b). The gain errors for SVT 5 are given in Fig. 4. In this case, a slight overshoot (resulting from greater series input inductance) was permitted to achieve some peaking in the frequency response. The equivalent SVT sampling rate used to obtain the step response data for both figures was $10 \mathrm{GHz}$. This sampling frequency, together with the pole at 600 or $700 \mathrm{MHz}$, gives upper bounds on the aliasing errors at $100 \mathrm{MHz}$ in Figs. 3 and 4 of approximately 0.3 percent [4]. Bounds on the aliasing errors at lower frequencies are proportionately smaller, giving for example, 0.03 percent at $10 \mathrm{MHz}$. 


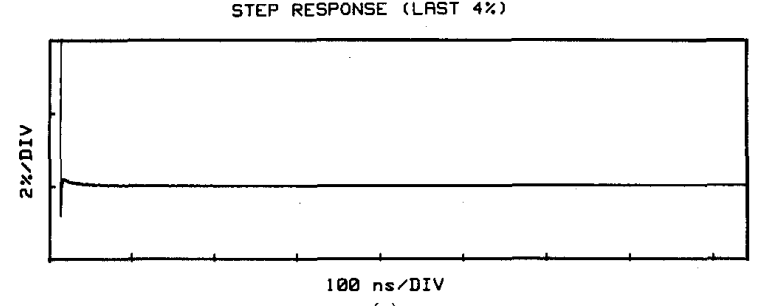

(a)

LOG FREQUENCY SPECTRUM

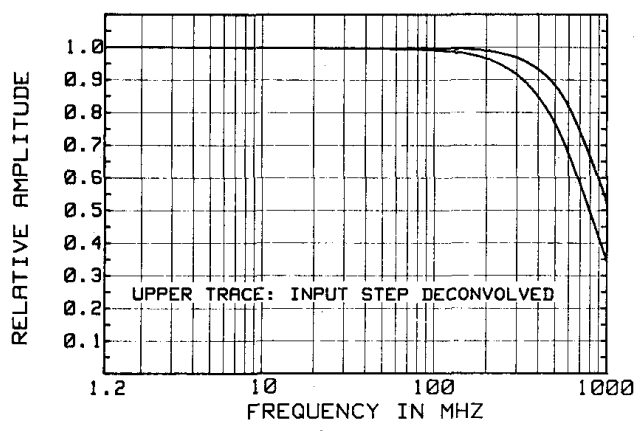

(b)

RELATIVE GAIN ERROR

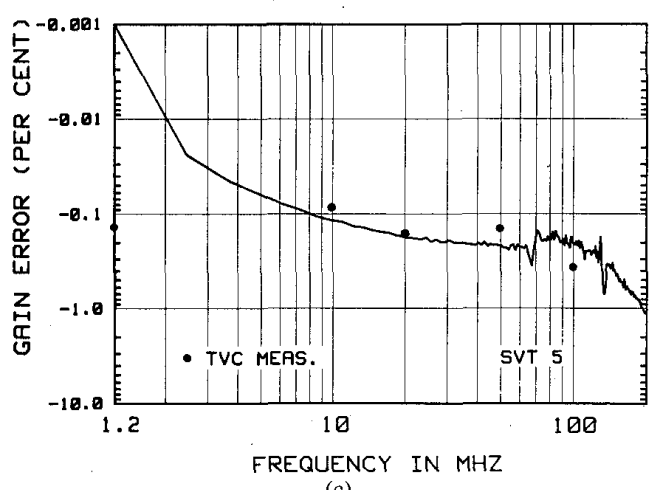

(c)

Fig. 4. Gain corrections from step response measurements, for SVT 5. (a) Showing last 4 percent of step response. (b) Frequency response computed from step response data. (c) Gain error relative to the first spectral line at $1.2 \mathrm{MHz}$.

Such gain correction computations are based on linear systems theory, and will be strictly valid only for systems which are linear. An SVT is expected to be essentially linear over much of its dynamic range; however, errors due to bias current for example, can be expected to cause superposition violations at low input levels where the bias current is proportionately greater. For sine wave inputs, the average value of the bias current has roughly the same functional relationship with sampling angle as does the input signal, and for large input signals its amplitude is independent of signal amplitude. The DVM is sensitive to the voltage drop across the impedance to ground (50 $\Omega$, not shown in Fig. 1) through which the bias current flows; therefore, an error voltage is introduced which is in-phase with the measured signal.

\section{Measurement Results and Error Sources}

Comparison measurements have been made using the SVT and calibrated thermal transfer instruments, over the frequency range of $1-100 \mathrm{MHz}$, at peak voltage levels of $1 \mathrm{~V}, 150$ and $15 \mathrm{mV}$. The $1-\mathrm{V}$ measurements were made with a thermal voltage converter (TVC) employing a 2.5$\mathrm{mA}$ UHF thermoelement. The test setup is shown in Fig. 5. The $150-$ and $15-\mathrm{mV}$ measurements were made with a micropotentiometer ( $\mu$ pot) using a $15-\mathrm{mA}$ UHF thermoelement and 10 - and $1-\Omega$ terminating disc resistors. This setup is shown in Fig. 6. The following is the measurement sequence for both setups.

1) With ac applied to the SVT and TVC, 144 samples are taken with the SVT over 4 complete cycles of the input waveform, i.e., one sample every $10^{\circ}$. From this data, the rms value is calculated. The sampling requires approximately $1 \mathrm{~s}$ per point.

2) The dc source is programmed to supply voltages equal to the rms voltage calculated in step 1).

3) The value of the thermocouple exponent $n$ is measured for the TVC.

4) The ac voltage is reapplied for $30 \mathrm{~s}$.

5) An ac-dc transfer is carried out using the sequence $+d c$, ac, $-d c$, with the dc voltages being read with the SVT. A settling time of $30 \mathrm{~s}$ is allowed after each step.

6) The sequence 1)-5) is repeated three times, giving a total of five sets of adjacent comparisons uniformly spaced in time. The average value of the five sets is relatively insensitive to linear drifts in the applied ac voltage.

Measurement results for the $1-\mathrm{V}$ level are plotted as solid circles in Figs. 3(c) and 4(c). These points represent the SVT gain error with respect to TVC measurements, for which calibration corrections have been applied. (These gain error measurements are relative to dc, rather than to the first ac spectral line of the plot.) The complete set of measurement results is listed in Table I. Here, the errors listed are the differences in percent between the voltages measured by the TVC (or $\mu$ pot) and the SVT. For these results, SVT gain error corrections (the solid curves in Figs. 3 and 4) have been applied, as have the TVC calibration corrections and corrections for transmission line errors as discussed below. The reported uncertainties associated with the TVC or $\mu$ pot calibrations are also given in the table. (The measurement results reported for $15 \mathrm{mV}$ were obtained with an uncalibrated $\mu$ pot, and therefore, represent only relative differences.)

\section{A. Time Base Errors}

It has been assumed to this point that the time sampling is perfect, i.e., that the sampling period $\Delta t$ is constant, and that exactly $k$ periods of the signal are sampled with a total of $n$ samples, where $k$ and $n$ are both integers. In practice, $\Delta t$ will have a random error component (time jitter), fixed pattern errors correlated with the sampling index, and a scale error such that $n \Delta t \neq k T$, where $T$ is the sine wave period. First, consider the scale error, which causes so-called truncation errors [6]. 


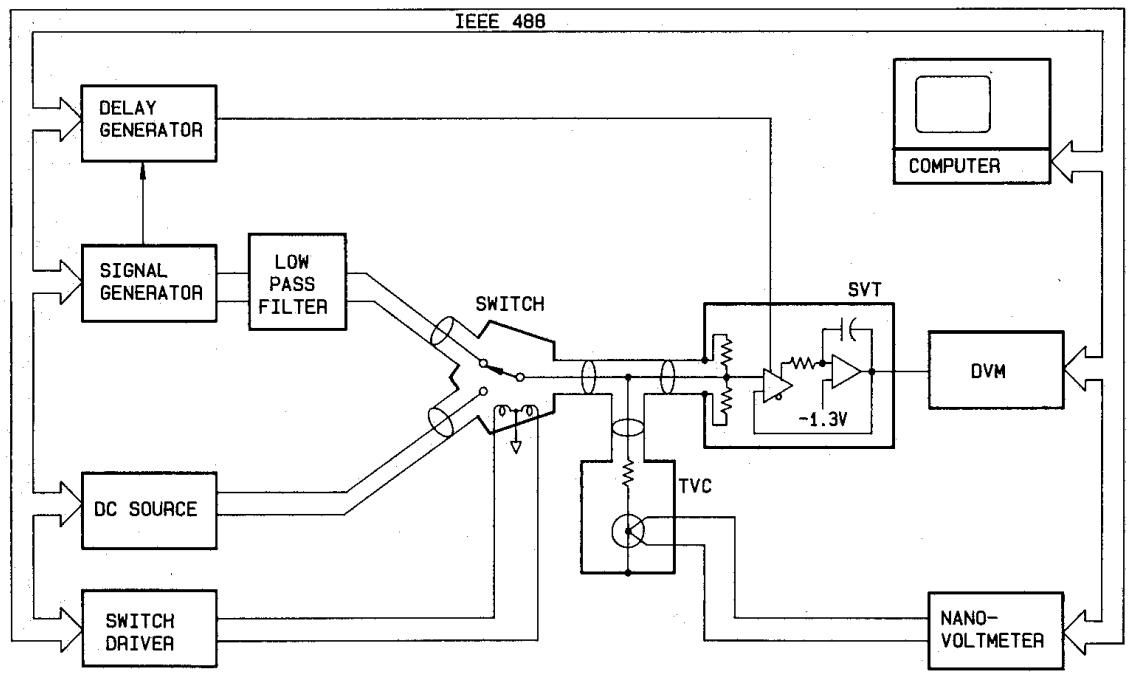

Fig. 5. Block diagram of system used in SVT-TVC comparison at the 1-V level.

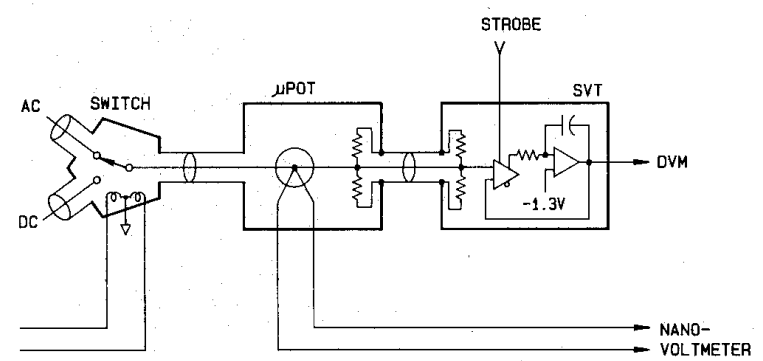

Fig. 6. Block diagram showing interconnection of the $\mu$ pot and SVT for comparison measurements at $15-$ and $150-\mathrm{mV}$ levels. The remainder of the system is the same as in Fig. 5 .

TABLE I

MEasured ERrors (\%) (TVC-SVT)

\begin{tabular}{|c|c|c|c|c|}
\hline frequency & $\begin{array}{l}1 \mathrm{v} \text { (SVT 4) } \\
\text { error }(\Delta \epsilon) \text {. }\end{array}$ & $\begin{array}{l}1 \vee \text { (SVT 5) } \\
\text { error }(\Delta t)\end{array}$ & $\begin{array}{c}150 \mathrm{mV} \\
\text { error }\end{array}\left(\begin{array}{l}\text { (SVT } \\
(\Delta \epsilon)\end{array}\right)$ & $\begin{array}{c}15 \mathrm{mV} \text { (SVT 4) } \\
\text { error }\end{array}$ \\
\hline $1.0 \mathrm{MHz}$ & $-0.01 \cdot(0.05)$ & $+0.13(0.05)$ & $+0.35(0.20)$ & +1.05 \\
\hline 10 & $0.00(0.10)$ & $-0.04(0.10)$ & $-0.03(0.30)$ & +1.30 \\
\hline 20 & $-0.04(0.20)$ & $-0.03(0.20)$ & $-0.01(0.30)$ & +1.30 \\
\hline 50 & $-0.19(0.50)$ & $-0.14(0.50)$ & $+0.72(0.50)$ & +0.95 \\
\hline 100 & $-1.35(1.00)$ & $-0.13(1,00)$ & $+1.03(1.10)$ & +0.54 \\
\hline
\end{tabular}

Define a sampled sine wave of period $T$ by

$$
V_{i}=V_{p} \sin (i \gamma+\alpha)
$$

where $\alpha$ is the starting phase of the sampling, $\gamma=$ $2 \pi \Delta t / T$, and $i$ is the sample index. The mean squared value of $n$ samples of $V_{i}$ is

$$
V^{2}=\left[(1 / n) \sum_{i=0}^{n-1} V_{i}^{2}\right]
$$

which can be rewritten [6] as

$$
V^{2}=\frac{V_{p}^{2}}{2}\left(1-\frac{1}{n} \sum_{0}^{n-1} \cos (2 i \gamma+2 \alpha)\right) \text {. }
$$

The first term of (3) represents the true mean squared value of the sine wave (defined over an integral number of periods); therefore, the second term represents the error $E$, resulting from sampling a nonintegral number of periods. Note that this error term vanishes for $n=k T / \Delta t$, where $k$ is an integer representing the number of sine wave periods sampled.

Define as in [6], the truncation angle $\delta$ to be the difference between the summation interval, given by $n \gamma$, and the nearest integral number of cycles $c$ of the sine wave, i.e., $\delta \equiv 2 \pi c-n \gamma$. Then the truncation error relative to the true mean squared value can be represented as

$$
\frac{1}{n} \cdot \frac{\sin \delta}{\sin \gamma} \cos (2 \alpha-\delta-\gamma)
$$

and assuming this error is small, the error relative to the root mean squared value is one half this error. For this work, measurements were made with a starting angle of $45^{\circ}$. With $\gamma$ typically $10^{\circ}$ and $\delta \leq 1^{\circ}$, (4) gives a truncation error (in the computed rms value) of $\leq 65 \mathrm{ppm}$ for $n=144$. Sampling under the same conditions with a starting angle of $0^{\circ}$ would give a truncation error of 345 ppm.

Variations of $\Delta t$ with time or with sampling index, $i$, are considered next. The SVT provides an estimate of each instantaneous value on a waveform based on a very large number of observations over as many repetitions of the waveform. It is offered here without proof (but will be documented in a later paper) that the estimate is essentially unbiased even though the observations are influenced by time jitter. (This is not the case for conventional 
equivalent time sampling systems in which the estimator is simply the average value of the observations [7].) Variations of $\Delta t$ with $i$, i.e., frequency modulation of the input sine wave, will, in the limit as $n$ becomes large, cause negligible error provided that the variations are uncorrelated with the input signal, and the mean of the variations is zero over the sampling interval. (A nonzero mean implies a scale error as discussed above.) This assertion follows from the fact that frequency modulation is a constant power process, i.e., the power of the modulated wave does not change as the degree of modulation changes [8]. An upper bound can be obtained for errors which are correlated with the input sine wave by considering the worstcase in which the time base errors, $\tau(i)$, are described by

$$
\tau(i)=\operatorname{sign}\{\sin (2 i \gamma+\alpha)\} \cdot \tau_{\max }
$$

where sign $\{\cdot\}$ indicates the sign of the bracketed function, $\tau_{\max }$ is the absolute value of the maximum estimated time base error, and $\gamma$ and $\alpha$ are as in (1). The error in the computed rms value of the sampled sine wave, due to errors $\tau(i)$, can be shown to be approximately $4 f \tau_{\max }$, where $f$ is the signal frequency, assuming $2 \pi f \tau \ll 1$. A $\tau_{\max }$ of $10 \mathrm{ps}$ [3] gives a limiting error in the rms value of 0.4 percent at $100 \mathrm{MHz}$ when the time base errors are all equal to $\pm \tau_{\max }$ and the signs are all correlated to give the worst error. For less pathological distributions, the resulting error should be much smaller.

\section{B. Transmission Line Errors}

Referring to Fig. 5 , it can be seen that a $50-\Omega$ coaxial tee coupling was used to connect the TVC and SVT to the voltage source. While the SVT had an input termination of $50 \Omega$ to match the characteristic impedance of the line, the TVC's input impedance was much higher $(\simeq 300 \Omega)$, causing a voltage difference (due to standing waves) between the TVC's input terminal and the common junction within the tee connector. Since the TVC had previously been calibrated with a voltage reference plane defined at the tip of the input connector, this difference had to be accounted for in the comparison measurements. The following correction was calculated.

Let $V_{1}$ be the voltage at the common junction within the tee, and $V_{2}$ be the voltage at the tip of the TVC input connector. From [9], for lossless lines we have

$$
V_{1}=V_{2} \cos \left(2 \pi \frac{\ell}{\lambda}\right)+j I_{r}(L / C)^{1 / 2} \sin \left(2 \pi \frac{\ell}{\lambda}\right)
$$

where $\ell$ is the distance between the tee junction and the TVC input connector, $\lambda$ is the wavelength of the input signal in the line, $I_{r}$ is the current at the TVC input connector, and $(L / C)^{1 / 2}$ is the characteristic impedance of the line, $Z_{0}$. Substituting $V_{2} / Z_{L}$ for $I_{r}$, where $Z_{L}$ is the impedance looking into the TVC (assumed to be resistive), $\beta$ for $2 \pi \ell / \lambda$, and $M$ for $Z_{0} / Z_{L}$, we have

$$
V_{1}=V_{2} \cos \beta+j V_{2} M \sin \beta
$$

and the magnitude of $V_{1} / V_{2}$ is given by

$$
V_{1} / V_{2}=\left[\cos ^{2} \beta+M^{2} \sin ^{2} \beta\right]^{1 / 2} .
$$

For $\beta \ll 1$, this becomes

$$
V_{1} / V_{2} \simeq 1-\frac{\beta^{2}}{2}\left(1-M^{2}\right) .
$$

At $100 \mathrm{MHz}$, the correction term was approximately 0.14 percent for the $2.5-\mathrm{cm}$ separation $(\ell)$ between the tee junction and the TVC input. In contrast, note that a mismatch of $1 \Omega$ between the SVT terminating resistor and the characteristic impedance of the line causes an error of only $50 \mathrm{ppm}$ for the same separating distance.

Tranmission line errors for the low voltage measurements were considered to be negligible since the $\mu$ pot was calibrated at its output connector with a $50-\Omega$ load, and the SVT was mated directed to it.

\section{Conclusion}

The RF voltage measurement capability of a SVT has been studied over the frequency range of $1-100 \mathrm{MHz}$, with applied voltages from $15 \mathrm{mV}$ to $1 \mathrm{~V}$. A simple method for software-correcting the gain errors based on step response data is presented, and an analysis of the effects of time base errors is given.

Comparison measurements made with thermal transfer instruments show differences at the $1-\mathrm{V}$ and $150-\mathrm{mV}$ levels which are generally within the limits of uncertainty established for the TVC or $\mu$ pot calibration services at NBS. These findings are consistent with other results reported in [10] which suggest that the reported uncertainties used in the NBS service are conservative. These uncertainty limits were exceeded in four out of 15 measurements reported in Table I. (The largest discrepancy was 0.13 percent versus a TVC calibration uncertainty of 0.05 percent, for SVT 5 at $1 \mathrm{MHz}$. Although the exact mechanism is unclear, it is likely that the larger "thermal tails" exhibited by SVT 5 are responsible for this discrepancy.)

At $15 \mathrm{mV}$, the differences between measurements made with the SVT and a $\mu$ pot were within 1.3 percent over the full frequency range. The lack of any strong dependence on frequency suggests that the major source of these differences may be bias current, as discussed at the end of Section III.

Based on the results reported in Table I, it appears that the SVT is capable of making RF voltage measurements with accuracies comparable to those of TVC's. In addition, the SVT offers much wider dynamic range, and can directly characterize arbitrary, repetitive waveforms in the time domain. As better comparators become available, further improvement in SVT performance can be expected.

\section{ACKNOWLEDGMENT}

The authors are indebted to $G$. Rebuldela for providing calibrations of the TVC and the $\mu$ pot, and to D. Flach for 
valuable discussions concerning the analysis of time base errors.

\section{REFERENCES}

[1] S. P. McCabe III; "A sampling voltage tracker for analyzing high speed waveforms," M.S. thesis, Univ. CA, Los Angeles, 1975.

[2] J. Halpert and M. Koen, "A waveform digitizer for dynamic testing of high speed data conversion components," in Dig. 1983 lnt. Test Conf., Philadelphia, PA, Oct. 1983.

[3] T. M. Souders, H. K. Schoenwetter, and P. S. Hetrick, "Characterization of a sampling voltage tracker for measuring fast, repetitive signals," IEEE Trans. Instrum. Meas., vol. IM-36, pp. 956-960, Dec. 1987.

[4] T. M. Souders and D. R. Flach, "Accurate frequency response de- terminations from discrete step response data," IEEE Trans. Instrum. Meas, vol. IM-36, pp. 433-439, June 1987.

[5] J. R. Andrews et al., "Reference waveform flat pulse generator," IEEE Trans. Instrum. Meas., vol. IM-32, pp. 27-32, Mar. 1983.

[6] G. N. Stenbakken et al., "NBS wideband sampling wattmeter," NBS Tech. Note 1221, May 1987.

[7] W. L. Gans, "The measurement and deconvolution of time jitter in equivalent-time waveform samplers,"' IEEE Trans. Instrum. Meas., vol. IM-32, pp. 126-133, Mar. 1983.

[8] H. S. Black, Modulation Theory. New York: Nostrand, 1953, p. 190.

[9] F. E. Terman, Radio Engineers Handbook, New York: McGrawHill, 1943, p. 185

[10] J. R. Kinard and C. Ti-Xiong, "Determination of ac-dc difference in the $0.1-100 \mathrm{MHz}$ frequency range," IEEE Trans. Instrum. Meas., pp. $360-367$, this issue. 